\title{
Полупроводниковые сплавы Гейслера на основе $\mathrm{Fe}$
}

\author{
() В.В. Ховайло ${ }^{1}$, А.И. Воронин ${ }^{1,3}$, В.Ю. Зуева ${ }^{1}$, М.А. Середина ${ }^{1}$, Р. Чаттерджи ${ }^{2}$ \\ ${ }^{1}$ Национальный исследовательский технологический университет „МИСиС“, \\ 119049 Москва, Россия \\ ${ }^{2}$ Indian Institute of Technology Delhi, \\ 110016 New Delhi, India \\ ${ }^{3}$ Южно-Уральский государственный университет (национальный исследовательский университет), \\ 454080 Челябинск, Россия \\ E-mail: khovaylo@misis.ru
}

(Получена 12 декабря 2016 г. Принята к печати 19 декабря 2016 г.)

\begin{abstract}
Дан краткий обзор по современному состоянию исследований полупроводниковых сплавов Гейслера на основе Fe. Наиболее значимым достижением в этой области является повышение термоэлектрической добротности до $Z T>1$ в соединениях $p$-типа проводимости на основе $\mathrm{Fe}(\mathrm{V}, \mathrm{Nb}) \mathrm{Sb}$. Наряду с этим соединением растущее внимание уделялось в последние годы исследованию перспективных термоэлектрических материалов на основе сплавов Гейслера $\mathrm{Fe}_{2} \mathrm{TiZ}(\mathrm{Z}=\mathrm{Al}, \mathrm{Si}, \mathrm{Sn})$ и изучению многофункциональных соединений $\mathrm{Fe}_{2} \mathrm{MnZ}(\mathrm{Z}=\mathrm{Al}, \mathrm{Si})$, которые могут представлять интерес и как термоэлектрические материалы, и как магнитные полупроводники с высокой температурой Кюри.
\end{abstract}

DOI: 10.21883/FTP.2017.06.44550.09

\section{1. Введение}

Полупроводниковые сплавы Гейслера имеют почти полувековую историю. Впервые их синтезировали в 1970 г. [1], но первые исследования зонной структуры и транспортных свойств были проведены спустя почти 20 лет [2]. До настоящего времени наиболее активно исследуемыми полупроводниковыми сплавами Гейслера являются соединения на основе $\mathrm{MNiSn}$ и $\mathrm{MCoSb}$ $(\mathrm{M}=\mathrm{Ti}, \mathrm{Zr}, \mathrm{Hf})$ со структурой типа $C 1_{b}$ (так называемые полу-Гейслеровы сплавы, half-Heusler alloys), которые считаются перспективными термоэлектрическими материалами для практических применений при температурах $700 \mathrm{~K}$ и выше. Как правило, сплавы Гейслера являются узкозонными полупроводниками, имеют большой коэффициент Зеебека $S$ (от -200 до -400 мкВ/К для соединений MNiSn) и высокие значения электрической проводимости $\sigma$. Основным недостатком MNiSn и $\mathrm{MCoSb}$ является их сравнительно большая теплопроводность $\kappa$ (до $10 \mathrm{BT} \cdot \mathrm{M}^{-1} \cdot \mathrm{K}^{-1}$ при комнатной температуре). На протяжении последних двух десятилетий основные усилия были направлены на понижение теплопроводности $\kappa$ этих материалов при сохранении большого коэффициента термоэлектрической мощности $S^{2} \sigma$. Для соединений $n$-типа проводимости на основе MNiSn ряд исследовательских групп сообщили о достижении значений термоэлектрической добротности $Z T \sim 1$, в то время как значения $Z T$ для соединений $p$-типа на основе $\mathrm{MCoSb}$ удалось повысить только до $\sim 0.5$ (см. обзорные работы [3-7]).

До последнего времени среди представителей полупроводниковых сплавов Гейслера со структурой типа $L 2_{1}$ (так называемые полные сплавы Гейслера, full Heusler alloys) наибольшее внимание с точки зрения термоэлектрических свойств привлекал сплав $\mathrm{Fe}_{2} \mathrm{VAl}$. Несмотря на сравнительно небольшие значения $Z T$, которые не превышают 0.3 в соединениях как $n$-типа, так и $p$-типа проводимости, исследованию $\mathrm{Fe}_{2} \mathrm{VAl}$ уделяется значительное внимание ввиду дешевизны термоэлектрических материалов на основе этого сплава. Так, в 2007 г. японские исследователи сообщили о разработке термоэлектрического модуля на основе $\mathrm{Fe}_{2} \mathrm{VAl}$ для рекуперации тепловой энергии выхлопных газов [8].

Наряду с термоэлектрическими материалами на основе сплавов MNiSn, MCoSb и $\mathrm{Fe}_{2} \mathrm{VAl}$ в последние годы ведутся интенсивные экспериментальные и теоретические исследования других сплавов Гейслера с перспективными термоэлектрическими свойствами. Экспериментальные работы увенчались разработкой материалов $p$-типа проводимости с $Z T \approx 1$ на основе сплавов Гейслера $\mathrm{FeRSb}(\mathrm{R}=\mathrm{V}, \mathrm{Nb})$, а теоретические исследования позволили выявить несколько полных сплавов Гейслера на основе железа, которые заслуживают экспериментального изучения их термоэлектрических свойств. В этой статье дан краткий обзор недавних работ по полупроводниковым сплавам Гейслера на основе железа.

\section{2. Сплавы Гейслера FeRSb $(R=\mathrm{V}, \mathrm{Nb})$}

Первые систематические исследования сплавов $\mathrm{FeRSb}$ показали [9], что они имеют тривиальные термоэлектрические свойства. Так, коэффициент Зеебека $S$, измеренный в интервале температур $T=240-500 \mathrm{~K}$, не превышал -80 мкВ/K в полупроводнике $n$-типа проводимости FeVSb и был практически равен нулю в $\mathrm{FeNbSb}$. Поскольку к тому же $\mathrm{FeNbSb}$ характеризуется сравнительно большим электрическим сопротивлением $(\rho \approx 140 \mathrm{мOм} \cdot$ см при $T=300 \mathrm{~K})$, было предположено [9], что FeNbSb является компенсированным полупроводником. Несмотря на то что и $S$, и $\rho$ оказались очень чувствительны к допированию $[9,10]$, высокая теплопроводность этих материалов $\left(\sim 15 \mathrm{BT} \cdot \mathrm{M}^{1} \cdot \mathrm{K}^{-1}\right.$ 
при $T=300 \mathrm{~K})$ не позволила авторам упомянутых работ достичь существенных значений $Z T$. Значительный прогресс в увеличении $Z T$ этих материалов был достигнут в работе [11], авторы которой показали, что увеличение содержания ванадия в FeVSb позволяет повысить коэффициент Зеебека до -175 мкВ/K, а метод механического сплавления приводит к заметному понижению теплопроводности. В результате этого было достигнуто $Z T \approx 0.31$ в образце $\mathrm{FeV}_{1.15} \mathrm{Sb}$ при $T=573 \mathrm{~K}$ [11]. Похожие значения ZT были получены также для стехиометрического $\mathrm{FeVSb}$, изготовленного методом левитационной плавки с последующим измельчением слитка и консолидации полученного порошка методом искрового плазменного спекания [12]. Опираясь на хорошо известный факт, что в твердых растворах сплавов и соединений замещение ,легких“ атомов с малым радиусом „тяжелыми“ атомами с большим радиусом приводит к возникновению локальных напряжений кристаллической решетки и, как следствие, к возникновению дополнительных центров рассеяния фононов, авторы работы [13] изучили влияние замены $\mathrm{V}$ на $\mathrm{Nb}$ на теплопроводность $\mathrm{FeV}_{1-x} \mathrm{Nb}_{x} \mathrm{Sb}$. Оказалось, что такое замещение позво-
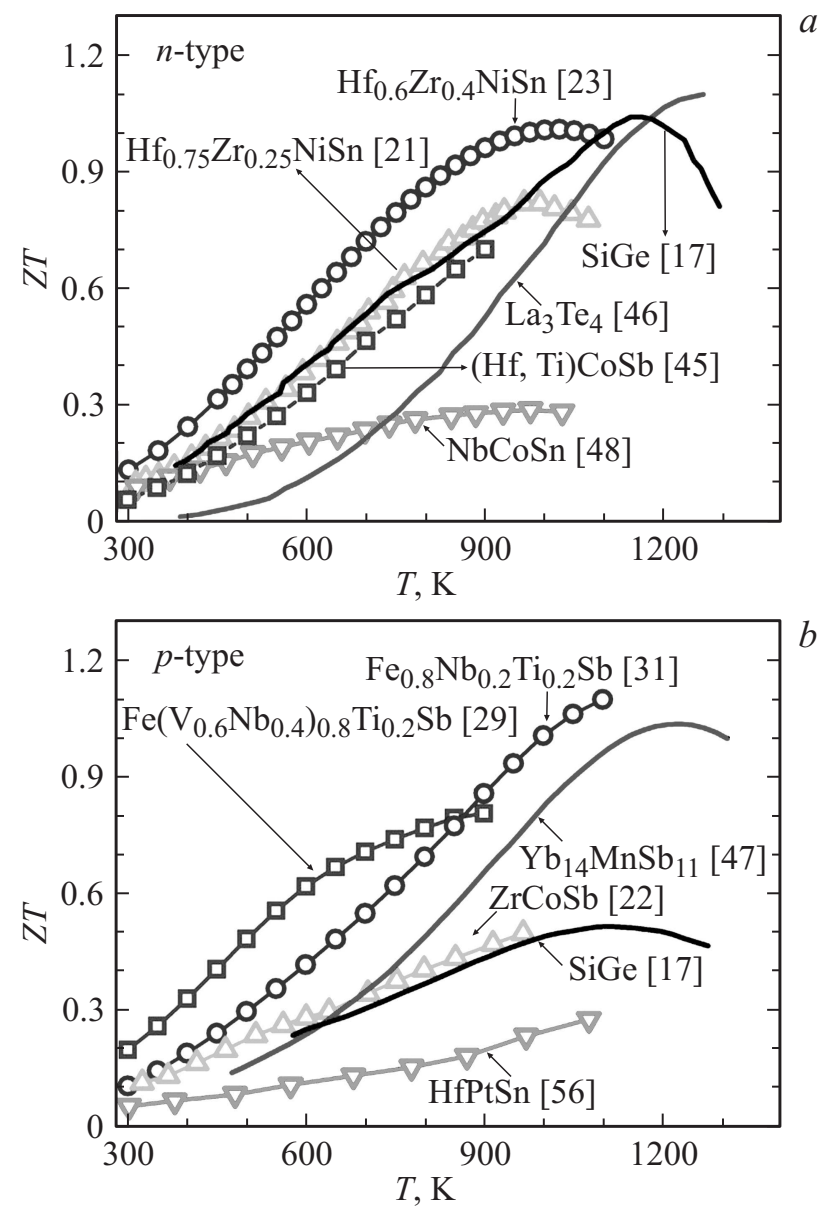

Рис. 1. $Z T$ высокотемпературных термоэлектрических материалов $n$ - и $p$-типа проводимости. По значениям $Z T$ сплавы Гейслера $n$-типа $[(\mathrm{Hf}, \mathrm{Zr}) \mathrm{NiSn}]$ и $p$-типа $[\mathrm{Fe}(\mathrm{Nb}, \mathrm{Ti}) \mathrm{Sb}]$ либо сравнимы, либо превосходят другие материалы [7]. ляет понизить теплопроводность до $\sim 5.5 \mathrm{BT} \cdot \mathrm{M}^{-1} \cdot \mathrm{K}^{-1}$ в образце $\mathrm{FeV}_{0.6} \mathrm{Nb}_{0.4} \mathrm{Sb}$, что в 2 раза меньше, чем в исходном FeVSb. Замена V на $\mathrm{Nb}$ приводит не только к понижению теплопроводности, но и к повышению электрического сопротивления $\mathrm{FeV}_{0.6} \mathrm{Nb}_{0.4} \mathrm{Sb}$, что в итоге негативно сказывается на ZT. Концентрация носителей заряда в этом соединении $n$-типа проводимости была оптимизирована за счет частичного замещения атомов железа атомами кобальта в $\mathrm{Fe}_{1-x} \mathrm{Co}_{x} \mathrm{~V}_{0.6} \mathrm{Nb}_{0.4} \mathrm{Sb}$, что позволило повысить $Z T$ до $\sim 0.33$ в образце с $x=0.015[14]$.

Очевидно, что повышение $Z T$ до $\sim 0.33$ в термоэлектрическом сплаве Гейслера $n$-типа представляет собой скорее фундаментальный интерес ввиду того, что в родственных полупроводниковых соединениях этого типа проводимости воспроизводимые значения $Z T$ достигают $\sim 1$ [4-7]. Основным фактором, который несомненно будет определять рост интереса как с фундаментальной, так и с практической точек зрения к сплавам $\mathrm{FeRSb}$, является то, что не только величина, но и знак коэффициента Зеебека в этих соединениях очень чувствительны к допированию [9]. Это позволило сначала достичь относительно большого для сплавов Гейслера $p$-типа проводимости значения $Z T \approx 0.43$ в $\mathrm{FeV}_{0.8} \mathrm{Ti}_{0.4} \mathrm{Sb}[15]$, которое вскоре было повышено до $Z T \geq 0.8$ в $\mathrm{Fe}\left(\mathrm{V}_{0.6} \mathrm{Nb}_{0.4}\right)_{1-x} \mathrm{Ti}_{x} \mathrm{Sb}[16]$ и до рекордных значений $Z T \geq 1$ в материалах на основе FeNbSb [17-19] (рис. 1) за счет комплексного допирования исходного соединения, направленного на оптимизацию зонной структуры FeNbSb [20].

\section{3. Сплавы Гейслера $\mathrm{Fe}_{2} \mathrm{YZ}(\mathrm{Y}=\mathrm{Ti}, \mathrm{Mn}$; $\mathbf{Z}=\mathbf{A l}, \mathbf{S i}, \mathbf{S n})$}

Наряду с системой сплавов $\mathrm{Fe}_{2} \mathrm{VAl}$, физическим свойствам которой посвящено множество работ, в последние годы наблюдается растущий интерес к полупроводниковым сплавам $\mathrm{Fe}_{2} \mathrm{TiZ}(\mathrm{Z}=\mathrm{Al}, \mathrm{Si}, \mathrm{Sn})$ и $\mathrm{Fe}_{2} \mathrm{MnZ}$ $(\mathrm{Z}=\mathrm{Al}, \mathrm{Si})$.

Экспериментальные исследования термоэлектрических свойств сплавов Гейслера $\mathrm{Fe}_{2} \mathrm{TiZ}(\mathrm{Z}=\mathrm{Al}, \mathrm{Sn})$ показали $[21,22]$, что коэффициент Зеебека в этих соединениях не превышает (по модулю) 50 мкВ/К. Кроме этого, большое количество дефектов упаковки, возникающих из-за атомного разупорядочения структурных подсистем $\mathrm{Fe}$ и $\mathrm{Ti}$, приводит к появлению локальных магнитоупорядоченных кластеров, что негативно сказывается на тепловых и транспортных характеристиках [23]. Теоретические расчеты [24] предсказывают, что большой, до -300 мкВ/К, коэффициент Зеебека может быть достигнут в сплавах $\mathrm{Fe}_{2} \mathrm{TiSn}_{1-x} \mathrm{Si}_{x}$ за счет изменения зонной структуры $\mathrm{Fe}_{2} \mathrm{TiSn}$, вызванной замещением олова кремнием. С целью проверки теоретических предсказаний нами была предпринята попытка синтеза поликристаллических образцов $\mathrm{Fe}_{2} \mathrm{TiSn}_{1-x} \mathrm{Si}_{x}$. Рентгеноструктурный анализ показал (рис. 2), что для двух крайних образцов $(x=0$ и 1$)$ параметр кристаллической решетки 


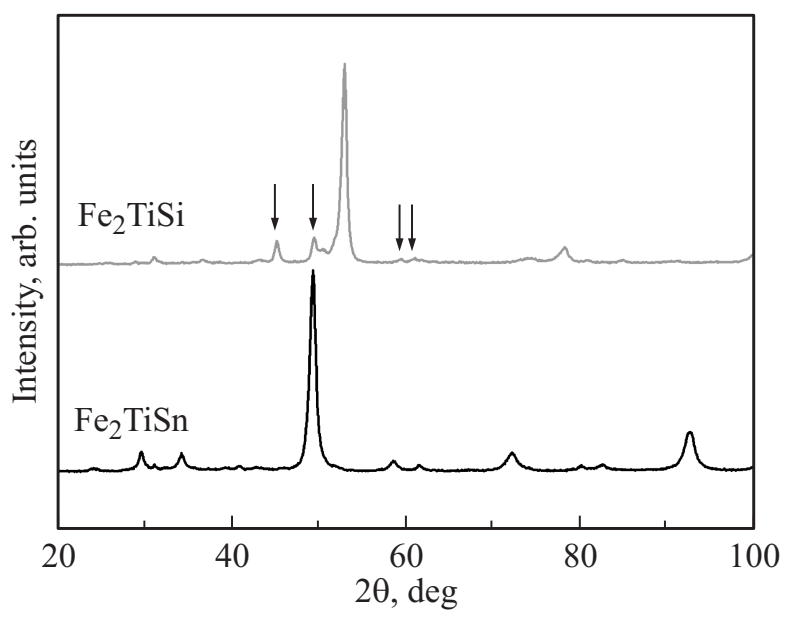

Рис. 2. Рентгенограммы образцов $\mathrm{Fe}_{2} \mathrm{TiSn}_{1-x} \mathrm{Si}_{x}$ с $x=0$ и 1 . Основной пик соответствует рефлексу (220) от кристаллической структуры Гейслера $L 2_{1}$. Стрелками отмечены рефлексы от примесной фазы [26].

и фазовый состав хорошо согласуются с литературными данными $[23,25]$. Что касается образцов промежуточных составов, то ни один из них не удалось получить в однофазном состоянии (см. [26]). Это, вероятно, обусловлено особенностями фазовых равновесий в тройной системе $\mathrm{Fe}-\mathrm{Ti}-\mathrm{Sn}$, в которой соединение $\mathrm{Fe}_{2} \mathrm{TiSn}$ становится нестабильным даже при малых отклонениях содержания $\mathrm{Sn}$ от стехиометрического [27].

В последние годы набирает популярность концепция „многофункциональных“ материалов, т.е. материалов, которые демонстрируют сразу несколько эффектов практической значимости. В этом контексте следует упомянуть магнитоупорядоченные сплавы Гейслера $\mathrm{Fe}_{2} \mathrm{MnZ}$, для которых теоретически предсказаны как привлекательные термоэлектрические свойства $[28,29]$, так и нетривиальные магнитотранспортные свойства, обусловленные особенностями строения электронной структуры [30,31]. Отметим, что в то время как теоретические предсказания существенных величин $Z T$ в сплавах Гейслера все еще ждут экспериментальных подтверждений, исследования халькопиритов $\mathrm{CuFeS}_{2}$ показали, что эти магнитные полупроводники действительно представляют интерес для дальнейшего изучения с точки зрения термоэлектрических свойств [32,33]. Если говорить об экспериментальных работах, в настоящее время основной интерес к соединениям на основе $\mathrm{Fe}_{2} \mathrm{MnZ}$ обусловлен возможностью практических применений их магнитных свойств. Несмотря на сравнительно низкие температуры Кюри исходных соединений $\left(T_{\mathrm{C}}=150 \mathrm{~K}\right.$ в $\mathrm{Fe}_{2} \mathrm{MnAl}$ и $T_{\mathrm{C}}=250 \mathrm{~K}$ в $\mathrm{Fe}_{2} \mathrm{MnSi}$, см. [34]), недавно было обнаружено, что частичное замещение железа никелем приводит к повышению $T_{\mathrm{C}}$ в $\mathrm{Fe}_{2} \mathrm{MnAl}$ до $>400 \mathrm{~K}$, при этом полупроводниковые свойства сохраняются [35]. Полупроводниковые свойства и высокую температуру Кюри $\left(T_{\mathrm{C}}=620 \mathrm{~K}\right)$ имеет также сплав на основе $\mathrm{Fe}_{2} \mathrm{MnSi}$, в котором половина атомов железа замещена кобальтом, (FeCo)MnSi [36]. Результаты этих исследований указывают на то, что материалы на основе $\mathrm{Fe}_{2} \mathrm{MnZ}$ могут представлять сушественный интерес с точки зрения применений в спинтронике.

\section{4. Заключение}

Основываясь на современном состоянии теоретических и экспериментальных исследований полупроводниковых сплавов Гейслера на основе Fe, можно выделить следующие основные тенденции и достижения. Во-первых, наблюдается растущий интерес к поиску и исследованию новых термоэлектрических материалов, которые отличаются дешевизной и не содержат токсичных элементов, и к разработке многофункциональных материалов, которые обладают и термоэлектрическими, и магнитными свойствами практической значимости. Во-вторых, учитывая, что в сплавах Гейслера $n$-типа проводимости (Ti,Zr,Hf)NiSn воспроизводимые значения термоэлектрической добротности достигают единицы, получение сплава Гейслера $p$-типа $\mathrm{Fe}(\mathrm{V}, \mathrm{Nb}) \mathrm{Sb}$ с $Z T>1$ позволяет надеяться на разработку и практическое применение высокоэффективных термоэлектрических генераторов, о прототипах которых уже сообщалось в литературе $[17,19]$.

Работа поддержана Российским научным фондом (грант № 16-42-02035).

\section{Список литературы}

[1] W. Jeitschko. Metall. Trans., 1, 3159 (1970).

[2] F.G. Aliev, N.B. Brandt, V.V. Moshchalkov, V.V. Kozyrkov, R.V. Skolozdra, A.I. Belogorokhov. Z. Phys. B, 75, 167 (1989).

[3] S.J. Poon. In: Recent Trends in Thermoelectric Materials Research II, ed. by T.M. Tritt [Semiconductors and Semimetals, v. 70 (Academic Press, 2001)].

[4] W. Xie, A. Weidenkaff, X. Tang, Q. Zhang, J. Poon, T.M. Tritt. Nanomaterials, 2, 379 (2012).

[5] S. Chen, Z. Ren. Mater. Today, 16, 387 (2013).

[6] J.-W.G. Bos, R.A. Downie. J. Phys.: Condens. Matter, 26 (43), 433201 (2014).

[7] T. Zhu, C. Fu, H. Xie, Y. Liu, X. Zhao. Adv. Energy Mater., 5 (19), 1500588 (2015).

[8] M. Mikami, K. Kobayashi, T. Kawada, K. Kubo, N. Uchiyama. J. Electron. Mater., 38, 1121 (2009).

[9] D.P. Young, P. Khalifah, R.J. Cava, A.P. Ramirez. J. Appl. Phys., 87, 317 (2000).

[10] L. Jodin, J. Tobola, P. Pecheur, H. Scherrer, S. Kaprzyk. Phys. Rev. B, 70 (18), 184207 (2004).

[11] M. Zou, J.-F. Li, P. Guo, T. Kita. J. Phys. D: Appl. Phys., 43 (41), 415403 (2010).

[12] C. Fu, H. Xie, Y. Liu, T.J. Zhu, J. Xie, X.B. Zhao. Intermetallics, 32, 39 (2013).

[13] C. Fu, H. Xie, T.J. Zhu, J. Xie, X.B. Zhao. J. Appl. Phys., 112 (12), 124915 (2012).

[14] C. Fu, Y. Liu, H. Xie, X. Liu, X. Zhao, G.J. Snyder, J. Xie, T. Zhu. J. Appl. Phys., 114 (13), 134905 (2013).

[15] M. Zou, J.-F. Li, T. Kita. J. Solid State Chem., 198, 125 (2013). 
[16] C. Fu, T. Zhu, Y. Pei, H. Xie, H. Wang, G.J. Snyder, Y. Liu, Y. Liu, X. Zhao. Adv. Energy Mater., 4 (18), 1400600 (2014).

[17] G. Joshi, R. He, M. Engber, G. Samsonidze, T. Pantha, E. Dahal, K. Dahal, J. Yang, Y. Lan, B. Kozinsky, Z. Ren. Energy Environ. Sci., 7, 4070 (2014).

[18] C. Fu, T. Zhu, Y. Liu, H. Xie, X. Zhao. Energy Environ. Sci., 8, 216 (2015).

[19] C. Fu, S. Bai, Y. Liu, Y. Tang, L. Chen, X. Zhao, T. Zhu. Nat. Commun., 6, 8144 (2015).

[20] X. Zhang, Y. Wang, Y. Yan, C. Wang, G. Zhang, Z. Cheng, F. Ren, H. Deng, J. Zhang. Sci. Rep., 6, 33120 (2016).

[21] R.O. Suzuki, T. Kyono. J. Alloys Comp., 377, 38 (2004).

[22] C.S. Lue, Y.-K. Kuo. J. Appl. Phys., 96, 2681 (2004).

[23] A. Slebarski, M.B. Maple, E.J. Freeman, C. Sirvent, D. Tworuszka, M. Orzechowska, A. Wrona, A. Jezierski, S. Chiuzbaian, M. Neumann. Phys. Rev. B, 62, 3296 (2000).

[24] S. Yabuuchi, M. Okamoto, A. Nishide, Y. Kurosaki, J. Hayakawa. Appl. Phys. Express, 6 (2), 025504 (2013).

[25] M. Meinert, M.P. Geisler, J. Schmalhorst, U. Heinzmann, E. Arenholz, W. Hetaba, M. Stöger-Pollach, A. Hütten, G. Reiss. Phys. Rev. B, 90 (8), 085127 (2014).

[26] В.Ю. Зуева, А.И. Воронин, Д.Ю. Карпенков, Х. Мики, В.В. Ховайло. ФТП (будет опубликовано).

[27] Y. Cai, Y. Wu, Z.Y. Xie, K. Llv, H.S. Liu, Z.P. Jin. Calphad, 49, 110 (2015).

[28] A.H. Reshak. RSC Adv., 4, 39565 (2014).

[29] T.M. Bhat, D.C. Gupta. RSC Adv., 6, 80302 (2016).

[30] Е.И. Шредер, А.Д. Свяжин, К.А. Фомина. ФММ, 113, 155 (2012).

[31] S. Sharma, S.K. Pandey. J. Magn. Magn. Mater., 403, 1 (2016).

[32] N. Tsujii, T. Mori. Appl. Phys. Express, 6 (4), 043001 (2013).

[33] R. Ang, A.U. Khan, N. Tsujii, K. Takai, R. Nakamura, T. Mori. Angew. Chem. Int. Ed., 54, 12909 (2015).

[34] Н.И. Коуров, В.В. Марченков, А.В. Королев, Л.А. Сташкова, С.М. Емельянова, Н.W. Weber. ФТТ, 57, 684 (2015).

[35] V. Khovaylo, M. Lyange, M. Seredina, R. Chatterjee, R. Varga. Proc. American Adv. Mater. Congress (Miami, USA, 2016) DOI: 10.5185/aamc.2016.

[36] L. Bainsla, A.I. Mallick, M. Manivel Raja, A.K. Nigam, B.S.D.Ch.S. Varaprasad, Y.K. Takahashi, A. Alam, K.G. Suresh, K. Hono. Phys. Rev. B, 91 (10), 104408 (2015).

Редактор Л.В. Шаронова

\section{Fe-based semiconducting Heusler alloys}

\author{
V.V. Khovaylo ${ }^{1}$, A.I. Voronin ${ }^{1,3}$, V.Yu. Zueva ${ }^{1}$, \\ M.A. Seredina ${ }^{1}$, R. Chatterdjee ${ }^{2}$ \\ ${ }^{1}$ National University of Science and Technology \\ „MISiS“, \\ 119049 Moscow, Russia \\ 2 Indian Institute of Technology Delhi, \\ 110016 New Delhi, India \\ ${ }^{3}$ National Research South Ural State University, \\ 454080 Chelyabinsk, Russia
}

Abstract A brief overview of the current state of researches on Fe-based semiconducting Heusler alloys is given. The most significant achievement in this area is the increase of thermoelectric figure of merit to $Z T>1$ in the $p$-type $\mathrm{Fe}(\mathrm{V}, \mathrm{Nb}) \mathrm{Sb}$-based compounds. Besides these compounds, growing attention has been paid in recent years to the study of promising thermoelectric materials based on $\mathrm{Fe}_{2} \mathrm{TiZ}(\mathrm{Z}=\mathrm{Al}, \mathrm{Si}, \mathrm{Sn})$ Heusler alloys and the investigation of multifunctional $\mathrm{Fe}_{2} \mathrm{MnZ}(\mathrm{Z}=\mathrm{Al}, \mathrm{Si})$ compounds, which may be of interest as thermoelectric materials as well as magnetic semiconductors with a high Curie temperature. 